\title{
GENERAL GYNECOLOGY
}

\section{Intravenous leiomyomatosis with intracardiac extension: a single-institution experience}

\author{
Micheal J. Worley Jr, MD; Anate Aelion, MD; Thomas A. Caputo, MD; Kenneth C. Kent, MD; Arash Salemi, MD; \\ Karl H. Krieger, MD; Michael J. Goldstein, MD; Dennis Y. Kuo, MD; Brian M. Slomovitz, MD
}

OBJECTIVE: The aim of this study was to outline the surgical management and outcomes for patients diagnosed with intravenous leiomyomatosis with intracardiac extension at a single institution.

STUDY DESIGN: This was a retrospective review of patients diagnosed with intravenous leiomyomatosis with intracardiac extension between 2002-2008.

RESULTS: Four patients were identified. The surgical approach in 3 (75\%) patients was a single-stage operation. Four (100\%) patients presented with cardiac symptoms: 3 (75\%) with syncope and 1 (25\%) with an abnormal electrocardiogram. Mean age at presentation was 48 years (range, 42-58 years). Complete resection of tumor was obtained in 1 (25\%) patient and 3 (75\%) patients experienced incomplete resection. Mean follow-up, including surveillance imaging, was 25.5 months (range, 8-57 months) and all 4 patients (100\%) are currently free of recurrence.

CONCLUSION: Surgical excision remains an effective therapy for treating patients with benign metastasizing leiomyomatosis. Incomplete surgical resection may result in favorable response.

Key words: cardiac mass, fibroid, intravenous leiomyomatosis

Cite this article as: Worley MJ Jr, Aelion A, Caputo TA, et al. Intravenous leiomyomatosis with intracardiac extension: a single-institution experience. Am J Obstet Gynecol 2009;201:574.e1-5.

I ntravenous leiomyomatosis is an uncommon manifestation of a benign tumor. Although this lesion is usually confined to the pelvis, the rare occurrence of intracardiac extension has been well documented. In 1907, Durck published the first account of intravenous leiomyomatosis with intracardiac extension from an autopsy analysis. ${ }^{1}$ Since then, at least 30 cases have been reported in the literature. There are currently 2 main theories regarding the cause of this neoplasm. The first suggests that the intima of myometrial sinuses is invaded by leiomyomatosis cells originating from the uterine myometrium. ${ }^{2}$ The second contends that the tumor is comprised of proliferating smooth muscle cells arising directly from the venous wall of the uterine or pelvic veins. ${ }^{3}$

Early diagnosis is difficult, because patients may be asymptomatic despite extensive intravenous extension. When symptoms are manifested, they are often secondary to direct cardiac involvement. These include dyspnea on exertion, shortness of breath, orthopnea, pleuritic chest pain, and/or syncopal episodes. Because of the rarity of intravenous leiomyomatosis, the aforementioned symptoms may lead to misdiagnosis and improper treatment. ${ }^{4}$

Since 2002, New York Presbyterian Hospital at the Weill Cornell Medical

From the Departments of Obstetrics and Gynecology (Drs Worley, Aelion, and Kuo) and Gynecologic Oncology (Dr Caputo) and the Divisions of Vascular Surgery (Dr Kent) and Cardiovascular Surgery (Drs Salemi and Krieger), Department of Surgery (Dr Goldstein), New York Presbyterian Hospital-Weill Cornell Medical College, New York, NY, and the Center for Liver Disease and Transplantation, New York Presbyterian Hospital, Columbia University, New York, NY; and the Department of Obstetrics and Gynecology, Division of Gynecologic Oncology (Dr Slomovitz), Women's Cancer Center, Morristown Memorial Hospital, Morristown, NJ.

Received April 21, 2009; revised May 10, 2009; accepted June 12, 2009.

Reprints not available from the authors.

Film footage of the surgical procedure described in this article has been used to create a video abstract and was presented at the 57th Annual Clinical Meeting of the American College of Obstetricians and Gynecologists, Chicago, IL, May 2-6, 2009.

0002-9378/\$36.00 • (c) 2009 Published by Mosby, Inc. • doi: 10.1016/j.ajog.2009.06.037

Center has successfully operated on and resected 4 cases of intravenous leiomyomatosis with intracardiac involvement. A review of Medline from 1975-2008 confirms that no other institution in the United States has had experience to this extent. The purpose of this retrospective review is to outline our experience and the surgical management of these rare lesions.

\section{Materials and Methods}

The medical records of all patients diagnosed with intravenous leiomyomatosis with intracardiac extension at New York Presbyterian Hospital-Weill Cornell Medical Center between 2002-2008 were reviewed. Institutional review board approval was obtained. Data were obtained from medical and pathologic records. Data collected included age, symptoms on presentation, race, extent of resection, postoperative length of stay, and surgical approach.

\section{Surgical technique}

Patient A was placed in the supine position and general endotracheal anesthesia was induced. A sternotomy and laparotomy were performed by the cardiothoracic and vascular surgery teams, respectively. Transesophageal echocar- 


\section{FIGURE 1 \\ Intracaval mass removal}

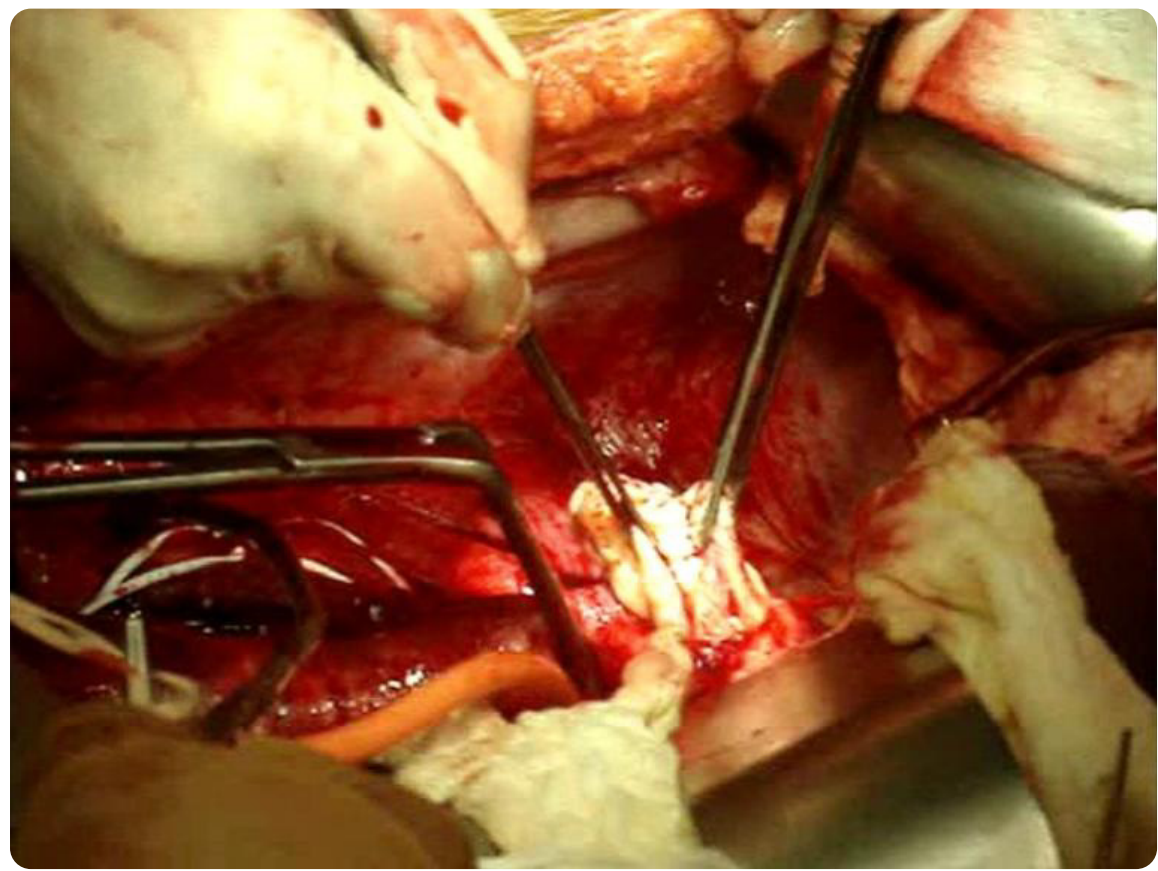

Surgical removal of mass from the inferior vena cava.

Worley. Intravenous leiomyomatosis. Am J Obstet Gynecol 2009.

\section{FIGURE 2}

\section{Intracaval mass specimen}

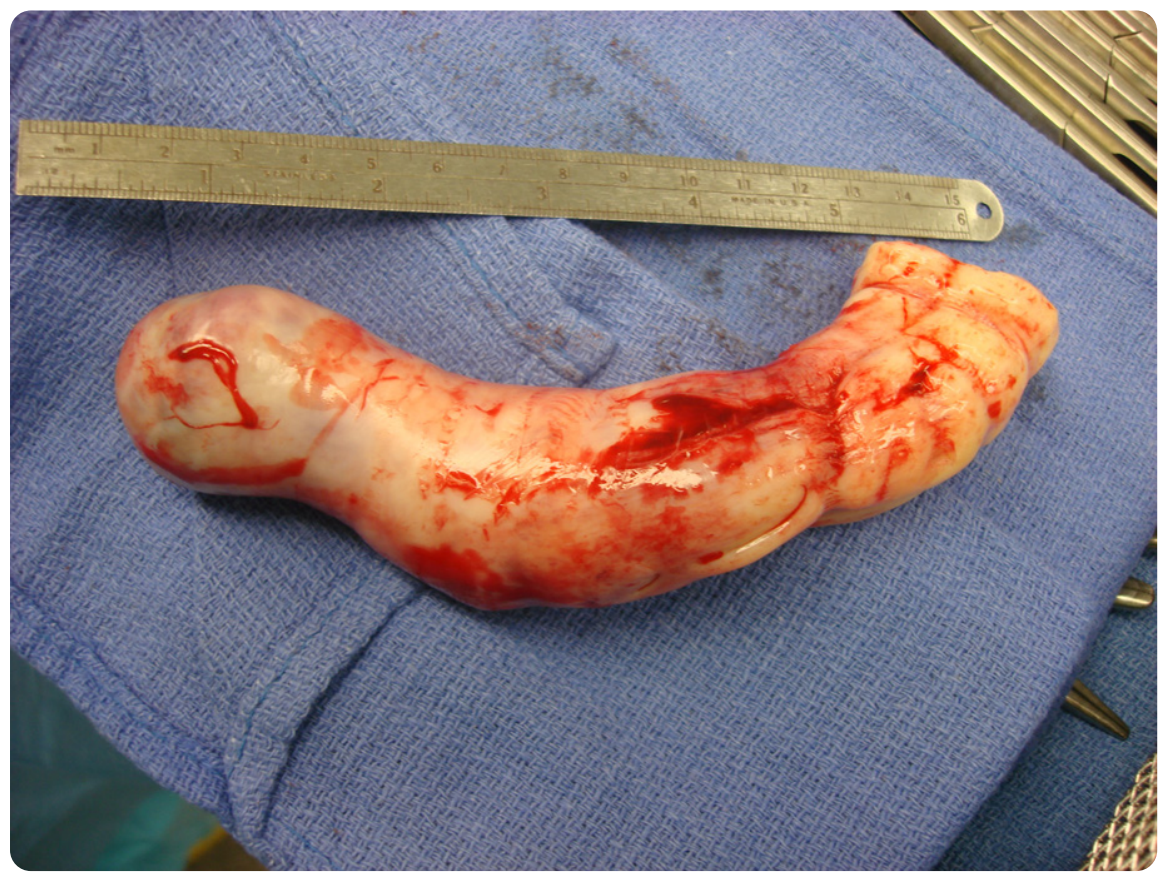

Gross surgical specimen of mass after removal from the inferior vena cava.

Worley. Intravenous leiomyomatosis. Am J Obstet Gynecol 2009. diography (TEE) revealed tumor within the right atrium. Medial visceral rotation, including hepatic mobilization, to obtain visualization of the inferior vena cava, was conducted by vascular and transplant surgery. Tumor within the inferior vena cava (IVC) was easily visualized. The IVC was dissected circumferentially and isolated by vascular surgery. After the right atrium was isolated by cardiothoracic surgery, a vessel loop was placed around the IVC, above the diaphragm. A longitudinal venotomy was made and the cephalad portion of the tumor was extracted (Figure 1). The tumor was visualized on echocardiography as it was removed. When this portion was completely removed, the venotomy was finger occluded, and the tumor was transected as far caudally as possible (Figure 2). The venotomy was oversewn and dissection and isolation of the IVC continued caudally. Full visualization of the IVC was difficult because of the myomatous uterus, so a supracervical hysterectomy and bilateral oophorectomy were conducted. With a similar technique as described, the tumor was removed from the caudal IVC, left common iliac vein, and left external iliac vein. As much tumor as possible was removed from the left internal iliac vein. The remaining tumor was isolated by ligating the proximal internal iliac vein with silk suture and hemostasis was confirmed before ending the procedure.

Patient $\mathrm{B}$ was brought to the operating room by the cardiothoracic surgery team. She was placed in the supine position, general endotracheal anesthesia was induced, and a median sternotomy was created. Intraoperative TEE revealed tumor within the right atrium, right ventricle, and potentially across the pulmonic valve. She was placed on cardiopulmonary bypass. An atriotomy was created and the tumor was retracted from the right ventricle and outflow tract. The caudal portion of the tumor was grasped as far caudally as possible and transected, allowing the tumor to retract into the abdominal IVC. The atriotomy was closed in 2 layers and the patient was separated from cardiopulmonary bypass. 
Two months later the patient returned to the operating room and a total abdominal hysterectomy with bilateral salpingo-oophorectomy was conducted. An attempt was made to place a filter above the level of the IVC to preclude tumor emboli. However, on fluoroscopic evaluation, the tumor appeared to have grown back into the right atrium. The remainder of the procedure was abandoned.

Eight days later, the patient returned to the operating room and was placed on cardiopulmonary bypass. The IVC was isolated and the tumor was removed from the IVC, right common iliac vein, and right internal iliac vein, as previously described. Again, residual tumor was isolated distally within the internal hypogastric vein.

Patient $\mathrm{C}$ was brought to the operating room 2 years after a total abdominal hysterectomy. She was placed in the supine position and general endotracheal anesthesia induced. In a similar fashion to patient $\mathrm{A}$, the tumor was removed from the right ventricle, right atrium, IVC, right common iliac vein, and right internal iliac vein. Residual tumor remained distally within the right internal iliac vein and was isolated in a fashion previously described. A bilateral salpingo-oophorectomy was then conducted by the gynecologic oncology service.

Patient D was brought to the operating room 5 years after a total abdominal hysterectomy with bilateral salpingo-oophorectomy. She was placed in the supine position, general endotracheal anesthetic was initiated, and she was placed on cardiopulmonary bypass. Isolation of the IVC was conducted, as previously described, and the tumor was removed from the right atrium, IVC, right common iliac vein, and right internal iliac vein, as previously described. A full dissection of the pelvic tributaries of the right internal iliac vein was conducted by the vascular and gynecologic oncology service. The entire pelvis was free of tumor on completion of the procedure.

\section{RESUlTS}

A total of 4 patients who had intravenous leiomyomatosis with intracardiac exten-

\section{FIGURE 3 \\ Intracardiac mass}

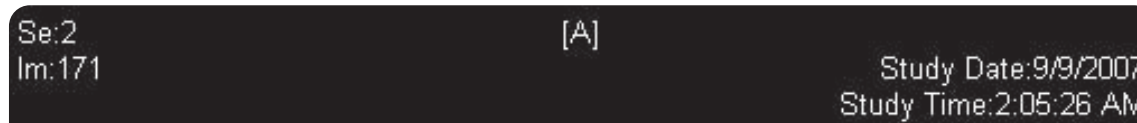

Study Time:2:05:26 AM

MRN:

[R]

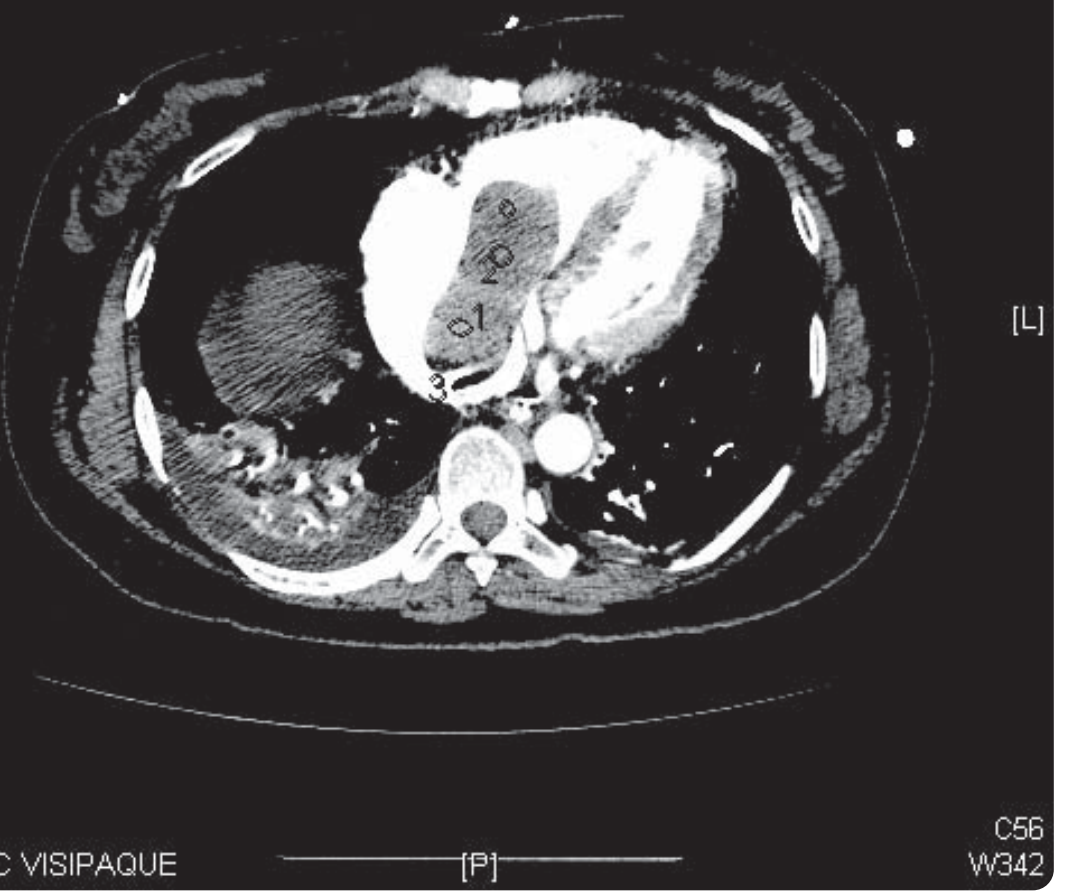

Computerized scan confirming right atrial involvement of leiomyoma.

Worley. Intravenous leiomyomatosis. Am J Obstet Gynecol 2009.

sion diagnosed were evaluated at New York Presbyterian Hospital-Weill Cornell Medical Center between 2002-2008. All patients underwent surgical treatment of their disease. The median patient age was 48 years (range, 42-58 years). Presenting symptoms in all $4 \mathrm{pa}$ tients were secondary to direct cardiac involvement (Figure 3). Three patients presented with syncope, and 1 patient presented with an abnormal electrocardiogram. There were 2 patients $(50 \%)$ in whom the tumor had extended into the right ventricle, and in the remaining 2 patients (50\%), the tumor extended into the right atrium. Two patients (50\%) had undergone a previous hysterectomy. Three patients $(75 \%)$ had successful resection in a 1-stage surgery. However, 1 patient managed with a single surgery had experienced an unsuccessful 2-stage approach, as previously described (Table 1).

Mean hospital stay after laparotomy was 12.75 days (range, $6-17$ days) and all 4 patients experienced an uncomplicated postoperative course. As mentioned previously, 2 patients underwent intracardiac resection before laparotomy and their hospital stays were 4 and 5 days, respectively. Complete resection of tumor was obtained in 1 (25\%) patient, and $3(75 \%)$ patients experienced incomplete resection. Mean follow-up, including surveillance imaging, was 25.5 months (range, 8-57 months), and all 4 patients (100\%) are currently free of recurrence (Table 2).

\section{Comment}

In our series, 3 of 4 patients were unable to undergo complete resection, with the 


\section{TABLE 1 \\ Descriptive charactersistics of patients with intravenous leiomyomatosis with intracardiac extension

\begin{tabular}{|c|c|c|c|c|c|c|c|}
\hline Patient & Age, y & Race & Presenting symptom & $\begin{array}{l}\text { Extent of cardiac } \\
\text { involvement }\end{array}$ & $\begin{array}{l}\text { Prior } \\
\text { hysterectomy }\end{array}$ & $\begin{array}{l}\text { Surgical } \\
\text { approach }\end{array}$ & $\begin{array}{l}\text { Cardiopulmonary } \\
\text { bypass }\end{array}$ \\
\hline A & 42 & African American & Abnormal EKG & Right atrium & No & 1-stage & No \\
\hline B & 45 & Asian & Syncope & Right atrium/right ventricle & No & 1 -stage ${ }^{a}$ & Yes $^{\mathrm{b}}$ \\
\hline C & 47 & Asian & Syncope & Right atrium/right ventricle & Yes & 1-stage & No \\
\hline D & 58 & African American & Syncope & Right atrium & No & 2-stage & $Y_{e s}{ }^{b}$ \\
\hline
\end{tabular} \\ EKG, electrocardiogram. \\ a Planned 2-stage approach. Atrial mass successfully removed. On fluroscopy at time of exploratory laparotomy, tumor was discovered to extend back into the right atrium. A 1 -stage removal of the intracaval tumor under cardiopulmonary bypass was performed 1 wk later; ${ }^{b}$ With gaining surgical experience after 2 surgeries, the need for cardiopulmonary bypass proved unnecessary. \\ Worley. Intravenous leiomyomatosis. Am J Obstet Gynecol 2009.}

remainder of tumor isolated distally within the deep tributaries of the hypogastric vessels. To date, all 4 patients in our series are currently free of disease progression. However, recurrent disease is common and can occur up to 15 years after surgery. Özer et $\mathrm{al}^{5}$ documented a 43-year-old woman who had undergone surgery to resect an intracaval and right atrial mass. After a symptom-free interval of 3 years, she presented to another medical center with abdominal pain, dizziness, and bilateral leg edema. Computerized tomography scan revealed the presence of a solid mass that extended from the common iliac vein into the right atrium. A subsequent biopsy confirmed the diagnosis of intravenous leiomyomatosis.

Redevelopment can also occur quite rapidly. In 2007, Esmaeilzadeh et al $^{6}$ described a 46-year-old woman who presented with several weeks of progressive dyspnea on exertion. She was subsequently diagnosed with intravenous leiomyomatosis with right ventricular involvement. Eight months before presentation, she had undergone cardiac surgery because of a right atrial mass that had been misdiagnosed as a myxoma. On reevaluation, the mass was reported to be a leiomyoma. The rapidity of recurrence in this case is similar to that of patient B. However, as displayed in Table 2, patient $B$ has experienced a disease-free interval of 4 years and 9 months from her 1-stage procedure.

Echocardiography, venography, chest and abdominal computed tomography imaging, and magnetic resonance venography aid in determining both the diagnosis and the extent of tumor involvement. In our series, a 1-stage operation was favored with the combined efforts of Gynecologic Oncology, Cardiothoracic, Transplant, and Vascular Surgery. Of the 4 patients we report, 3 underwent a 1-stage procedure. As mentioned previously, 1 patient underwent a 1-stage procedure after a failed 2-stage procedure. In the aforementioned case, the intracardiac mass was removed, and, during the attempted second surgery, the mass was found to have reextended into the right atrium.

Successful therapy relies on surgical resection, and, as our series displays, complete surgical excision is often precluded by involvement of the tumor within the deep tributaries of the hypogastric vessels. However, isolation of residual tumor appears equally important to prevent recurrences that can necessitate further surgery or lead to death. As these lesions are at great risk to recur, close long-term follow-up is essential. In our patients, postoperative pelvic ultrasonography was conducted every 3 months for the first year and biannually thereafter. The use of postoperative antiestrogens, such as tamoxifen, has been considered, but their efficacy has yet to be established. ${ }^{4}$ All 4 patients in our series were offered postoperative treatment with megesterol. One patient declined and

\section{TABLE 2}

\section{Postoperative characteristics of patients with intravenous leiomyomatosis with intracardiac extension who underwent surgical resection}

\begin{tabular}{|c|c|c|c|c|c|}
\hline Patient & Age, y & Surgical approach & $\begin{array}{l}\text { Postoperative length } \\
\text { of stay, d }\end{array}$ & $\begin{array}{l}\text { Complete } \\
\text { resection }\end{array}$ & Follow-up \\
\hline $\mathrm{A}$ & 42 & 1-stage & 14 & $\mathrm{No}^{\mathrm{a}}$ & 8 mo. No recurrence \\
\hline$B$ & 45 & 1-stage & $17^{\mathrm{b}}$ & $\mathrm{No}^{\mathrm{a}}$ & 4 y, 9 mo. No recurrence \\
\hline C & 47 & 1-stage & 14 & $\mathrm{No}^{\mathrm{a}}$ & $1 \mathrm{y}, 3 \mathrm{mo}$. No recurrence \\
\hline $\mathrm{D}$ & 58 & 2-stage & $6^{\mathrm{c}}$ & Yes & $1 \mathrm{y}, 10 \mathrm{mo}$. No recurrence \\
\hline
\end{tabular}


was placed on letrozole. Long-term efficacy comparing choices in postoperative treatment is lacking but presents an area of interest for future investigations.

\section{REFERENCES}

1. Durck H. Ueber ien Kontinvierlich durch die entere Holhlvene in das Herz vorwwachsendes: fibromyom des uterus. Munchen Med Wehnschr 1907;54:1154.

2. Sizenfrey A. Uber Venenmyome des Uterus mit Intravaskularem Wachstum. Z Beitr Geburtsh Gynaekol 1911;68:1.

3. Knauer E, Beitrag E. Zur anatomie der Uterusmyome. Z Beitr Geburtsh Gynaekol 1903; 1:696-735.

4. Nam MS, Jeon MJ, Kim YT, et al. Pelvic leiomyomatosis with intracaval and intracardiac extension: a case report and review of the literature. Gynecol Oncol 2003;89:175-80.

5. Özer N, Engin H, Akgül E, et al. An unusual case of recurrent mass in the right atrium: intravenous leiomyomatosis. Echocardiography 2005;22:514-6.

6. Esmaeilzadeh M, Tavakolli A, Safaei A. Recurrent intracardiac leiomyomatosis. Can J Cardiol 2007;23:1085-6. 